Published in final edited form as:

Lancet. 2014 January 4; 383(9911): 60-68. doi:10.1016/S0140-6736(13)61914-5.

\title{
Effect of an RNA interference drug on the synthesis of proprotein convertase subtilisin/kexin type 9 (PCSK9) and the concentration of serum LDL cholesterol in healthy volunteers: a randomised, single-blind, placebo-controlled, phase 1 trial
}

\author{
Kevin Fitzgerald, PhD, \\ Alnylam Pharmaceuticals, Cambridge, MA, USA \\ Maria Frank-Kamenetsky, PhD, \\ Alnylam Pharmaceuticals, Cambridge, MA, USA \\ Svetlana Shulga-Morskaya, MSc, \\ Alnylam Pharmaceuticals, Cambridge, MA, USA \\ Abigail Liebow, BS, \\ Alnylam Pharmaceuticals, Cambridge, MA, USA \\ Brian R Bettencourt, PhD, \\ Alnylam Pharmaceuticals, Cambridge, MA, USA \\ Jessica E Sutherland, PhD, \\ Alnylam Pharmaceuticals, Cambridge, MA, USA \\ Renta M Hutabarat, PhD, \\ Alnylam Pharmaceuticals, Cambridge, MA, USA \\ Valerie A Clausen, PhD, \\ Alnylam Pharmaceuticals, Cambridge, MA, USA \\ Verena Karsten, PhD, \\ Alnylam Pharmaceuticals, Cambridge, MA, USA \\ Jeffrey Cehelsky, MBA, \\ Alnylam Pharmaceuticals, Cambridge, MA, USA \\ Saraswathy V Nochur, PhD,
}

\footnotetext{
Correspondence to: Dr Kevin Fitzgerald, Alnylam Pharmaceuticals, Cambridge, MA 02446, USA kfitzgerald@alnylam.com. Contributors

All authors interpreted the data and collaborated in the preparation of the report. KF and AS had primary responsibility for the decision to submit for publication, and all authors vouch for the completeness and accuracy of the data and analyses. KF, MF-K, SS$\mathrm{M}, \mathrm{JH}, \mathrm{VKo}$, and $\mathrm{AL}$ were responsible for the preclinical data from non-human primates; RMH and VAC were responsible for the pharmacokinetic and pharmacodynamic data and figures; JES was responsible for toxicology data in support of the phase 1 trial; and KF, BRB, VKa, SVN, TM, JCe, JCh, JR, MM, AKV, JAG, and AS were responsible for the design and execution of the phase 1 trial. $\mathrm{BRB}, \mathrm{AS}, \mathrm{JCe}$, and VKa were responsible for collection and collation of the clinical data, and BRB was responsible for the statistical analysis.

Conflicts of interest

KF, SS-M, AL, BRB, JES, RMH, VAC, VKa, JCe, SVN, AKV, JAG, and AS are employees of Alnylam Pharmaceuticals with salary and stock options. VKo, MF-K, JH, MM, JR, JCh, and TM declare that they have no conflicts of interest.
} 
Alnylam Pharmaceuticals, Cambridge, MA, USA

Victor Kotelianski, MD,

Alnylam Pharmaceuticals, Cambridge, MA, USA

Jay Horton, MD,

Internal Medicine and Molecular Genetics, University of Texas South Western, Dallas, TX, USA

Timothy Mant, MD,

Quintiles Drug Research Unit at Guy's Hospital, London, UK

Joseph Chiesa, MD,

Covance Clinical Research Unit, Leeds, UK

James Ritter, MD,

Quintiles Drug Research Unit at Guy's Hospital, London, UK

Malathy Munisamy, MD,

Covance Clinical Research Unit, Leeds, UK

Akshay K Vaishnaw, MD,

Alnylam Pharmaceuticals, Cambridge, MA, USA

Jared A Gollob, MD, and

Alnylam Pharmaceuticals, Cambridge, MA, USA

Amy Simon, MD

Alnylam Pharmaceuticals, Cambridge, MA, USA

\section{Summary}

Background-Proprotein convertase subtilisin/kexin type 9 (PCSK9) binds to LDL receptors, leading to their degradation. Genetics studies have shown that loss-of-function mutations in PCSK9 result in reduced plasma LDL cholesterol and decreased risk of coronary heart disease. We aimed to investigate the safety and efficacy of ALN-PCS, a small interfering RNA that inhibits PCSK9 synthesis, in healthy volunteers with raised cholesterol who were not on lipid-lowering treatment.

Methods-We did a randomised, single-blind, placebo-controlled, phase 1 dose-escalation study in healthy adult volunteers with serum LDL cholesterol of $3.00 \mathrm{mmol} / \mathrm{L}$ or higher. Participants were randomly assigned in a 3:1 ratio by computer algorithm to receive one dose of intravenous ALN-PCS (with doses ranging from 0.015 to $0.400 \mathrm{mg} / \mathrm{kg}$ ) or placebo. The primary endpoint was safety and tolerability of ALN-PCS. Secondary endpoints were the pharmacokinetic characteristics of ALN-PCS and its pharmacodynamic effects on PCSK9 and LDL cholesterol. Study participants were masked to treatment assignment. Analysis was per protocol and we used ANCOVA to analyse pharmacodynamic endpoint data. This trial is registered with ClinicalTrials.gov, number NCT01437059.

Findings-Of 32 participants, 24 were randomly allocated to receive a single dose of ALN-PCS $(0.015 \mathrm{mg} / \mathrm{kg}$ [n=3], $0.045 \mathrm{mg} / \mathrm{kg}$ [n=3], $0.090 \mathrm{mg} / \mathrm{kg}$ [n=3], $0.150 \mathrm{mg} / \mathrm{kg}$ [n=3], 0.250 mg/kg [n=6], or $0.400 \mathrm{mg} / \mathrm{kg}[\mathrm{n}=6]$ ) and eight to placebo. The proportions of patients affected by treatment-emergent adverse events were similar in the ALN-PCS and placebo groups (19 [79\%] vs 
seven [88\%]). ALN-PCS was rapidly distributed, with peak concentration and area under the curve ( 0 to last measurement) increasing in a roughly dose-proportional way across the dose range tested. In the group given $0.400 \mathrm{mg} / \mathrm{kg}$ of ALN-PCS, treatment resulted in a mean $70 \%$ reduction in circulating PCSK9 plasma protein $(\mathrm{p}<0.0001)$ and a mean $40 \%$ reduction in LDL cholesterol from baseline relative to placebo $(\mathrm{p}<0.0001)$.

Interpretation-Our results suggest that inhibition of PCSK9 synthesis by RNA interference (RNAi) provides a potentially safe mechanism to reduce LDL cholesterol concentration in healthy individuals with raised cholesterol. These results support the further assessment of ALN-PCS in patients with hypercholesterolaemia, including those being treated with statins. This study is the first to show an RNAi drug being used to affect a clinically validated endpoint (ie, LDL cholesterol) in human beings.

Funding-Alnylam Pharmaceuticals.

\section{Introduction}

LDL cholesterol is one of the major risk factors for coronary heart disease, with a continuous and graded association between its plasma concentration and risk - for every $0.78 \mathrm{mmol} / \mathrm{L}(30 \mathrm{mg} / \mathrm{dL})$ change in LDL cholesterol, the relative risk for coronary heart disease changes by roughly $30 \% .^{1,2}$ Additionally, in a large meta-analysis ${ }^{3}$ of 21 statin studies, the investigators concluded that for every $1.01 \mathrm{mmol} / \mathrm{L}(39 \mathrm{mg} / \mathrm{dL})$ reduction in LDL cholesterol with statin treatment, cardiovascular events were reduced by about $22 \%{ }^{3}$ Despite the extensive use of statins, existing treatments for the management of raised LDL cholesterol remain inadequate. This is especially true for individuals with pre-existing coronary heart disease or diabetes, who are at the highest risk and require the most aggressive management of hyper-cholesterolaemia. ${ }^{4}$ Among high-risk individuals, it is estimated that only $50 \%$ achieve the target LDL cholesterol of less than $2.59 \mathrm{mmol} / \mathrm{L}$ at 6 months after statin treatment, despite close monitoring and optimisation of the drug regimen. ${ }^{5-9}$ With the LDL cholesterol target of less than $1.81 \mathrm{mmol} / \mathrm{L}$ in high-risk individuals, the number who reach their LDL cholesterol goals is even lower at 30\%.9,10 Thus, a clear unmet medical need exists for hypercholesterolaemia treatments, especially in high-risk patient populations.

Proprotein convertase subtilisin/kexin type 9 (PCSK9) is a member of the serine protease family and was first connected to cholesterol metabolism when gain-of-function mutations in PCSK9 were identified in people with familial hypercholesterolaemia who did not have mutations in the LDL receptor $(L D L R)$ or apolipoprotein B (APOB) genes. ${ }^{11}$ Animal studies subsequently determined that PCSK9 binds hepatocyte LDLRs both intracellularly and extracellularly, leading to their lysosomal degradation (figure 1). ${ }^{12,13}$ Loss-of-function mutations in PCSK9 have also been described in human beings, and are associated with reductions in LDL cholesterol and risk of coronary heart disease. ${ }^{14,15}$ Several individuals with no circulating PCSK9 due to compound heterozygous loss-of-function mutations have also been identified. These individuals have very low LDL cholesterol $(<0.52 \mathrm{mmol} / \mathrm{L})$, but are otherwise healthy. ${ }^{16,17}$ In loss-of-function mouse models for PCSK9, reductions in total cholesterol have been noted, ${ }^{18}$ consistent with the human phenotype. Collectively, these genetics studies support the hypothesis that lowering of circulating plasma PCSK9 by 
inhibiting its synthesis in hepatocytes should lower LDL cholesterol, potentially resulting in reduced risk of coronary heart disease. Additionally, some evidence suggests that statin treatment increases circulating plasma PCSK9, which could limit the effectiveness of statins as the dose is increased. ${ }^{19-22}$ Human clinical trials with PCSK9-blocking antibodies have shown significant reductions in LDL cholesterol in healthy volunteers ${ }^{23-24}$ and in individuals with hypercholesterolaemia, with and without statins. ${ }^{25-30}$

Small interfering RNA (siRNA) can direct sequence-specific degradation of messenger RNA, leading to suppression of synthesis of the corresponding proteins, as part of the natural biological process known as RNA interference (RNAi). ${ }^{31,32}$ Our group has previously reported the acute, hepatocyte-specific lowering of synthesis and plasma concentrations of PCSK9 by treatment with a PCSK9-specific siRNA formulated in a lipid nanoparticle in several preclinical models. ${ }^{33}$ This treatment resulted in substantial and durable lowering of LDL cholesterol, without an effect on HDL cholesterol. Additionally, we confirmed in these models that the reduction in PCSK9 was based on an RNAi mechanism and resulted in increased numbers of LDLRs on hepatocyte membranes. ${ }^{33} \mathrm{We}$ aimed to investigate the safety and efficacy in human beings of ALN-PCS, an siRNA that inhibits PCSK9 synthesis formulated in a novel lipid nanoparticle for delivery. ${ }^{34}$

\section{Methods}

\section{Study design and participants}

We undertook a randomised, single-blind, placebo-controlled, phase 1 clinical trial at two phase 1 units in the UK (Covance Clinical Research Unit, Leeds, and Quintiles Drug Research Unit at Guy's Hospital, London). Eligible participants were healthy adults aged 18-65 years with an LDL cholesterol higher than $3.00 \mathrm{mmol} / \mathrm{L}$. Inclusion criteria required that they had received no lipid-lowering treatments in the 30 days before screening and that they had fasting triglyceride con centrations of $2.8 \mathrm{mmol} / \mathrm{L}$ or less. Exclusion criteria included a body-mass index of $35 \mathrm{~kg} / \mathrm{m}^{2}$ or higher, use of dietary supplements known to affect lipids in the 4 weeks before screening, and a change in exercise regimen in the 4 weeks before screening.

We obtained written informed consent from all participants. The study protocol is available from the Alnylam website and includes the full list of inclusion and exclusion criteria. The protocol was approved by an ethics committee, and the study was done in accordance with the Good Clinical Practice guidelines and the Declaration of Helsinki. The trial was continued to completion.

\section{Randomisation and masking}

Treatment assignment was masked only from study participants, and not from those investigators who gave the interventions, those who assessed the outcomes, or those who analysed the data. At each site, the investigator enrolled volunteers, who were randomly assigned by the site's research pharmacist. Within each dose cohort of four volunteers, the first two eligible participants were assigned by a computer-generated, predetermined, randomisation algorithm, with one assigned to ALN-PCS and the other to placebo (normal 
saline). The remaining two participants of each cohort received ALN-PCS. Because ALNPCS is not identical in appearance to saline, the infusion sets (intravenous bag with drug and tubing) were covered so that the contents of the infusion could not be seen by the participants.

\section{Procedures}

The appendix (pp 9-12) summarises the study measurements used, and detailed methods are provided in the study protocol. All participants were admitted to the phase 1 unit 3 days before dosing and remained at the unit site for 4 days after dosing. During this time at the phase 1 unit, all participants received a standard diet designed to meet the nutritional requirements necessary to promote health and prevent diet-related diseases.

On the evening before and the morning of dosing, all participants received oral corticosteroids, histamine receptor $(\mathrm{H} 1$ and $\mathrm{H} 2)$ blockers, and paracetamol to reduce the potential for infusion-related reactions. Specifically, participants received dexamethasone ( 8 $\mathrm{mg}$ the evening before dosing and $20 \mathrm{mg} 60 \mathrm{~min}$ before the start of infusion of ALN-PCS or placebo) and paracetamol (500 mg orally the evening before dosing and again 30-60 min before the start of infusion). They also received an oral $\mathrm{H} 2$ blocker (ranitidine $150 \mathrm{mg}$ or famotidine $20 \mathrm{mg}$ ) and an $\mathrm{H} 1$ blocker (10 $\mathrm{mg}$ cetirizine) the evening before dosing and again 30-60 min before the start of infusion.

A single $1 \mathrm{~h}$ intravenous infusion of ALN-PCS or placebo (normal saline) was given to all participants. Six different doses $(0 \cdot 015,0.045,0.090,0 \cdot 150,0 \cdot 250$, and $0.400 \mathrm{mg} / \mathrm{kg})$ of ALN-PCS were assessed. Each dose cohort consisted of four participants randomly assigned in a 3:1 ratio of ALNPCS treatment to placebo. Two additional cohorts of four participants (randomly assigned in the same ratio) were given the 0.250 and $0.400 \mathrm{mg} / \mathrm{kg}$ doses of ALNPCS (or placebo). Dose was escalated after satisfactory review of all safety information by the safety review committee.

Participants were seen for prespecified follow-up visitsat various times during the 180 days after study drug dosing. Data for adverse events were collected for 28 days after dosing. Clinical laboratory safety tests, physical examinations, and medically important events were assessed up to day 180 . Safety reviews were done by a safety review committee between each ascending dose cohort before dose escalation. These reviews consisted of assessment of treatment-emergent adverse events, 12-lead electrocardiographs (ECGs), lead II ECG monitoring, arterial oxygen saturation $\left(\mathrm{SaO}_{2}\right)$ via pulse oximetry, vital signs (blood pressure, pulse, oral body temperature, and respiration rate), clinical laboratory safety tests (haematology, serum chemistry, coagulation parameters, urinalysis, cytokines, complement activation fragment $[\mathrm{Bb}]$, and troponin), and physical examinations.

Concentrations of interferon $\alpha$, interferon $\gamma$, inter-leukin 6 , interleukin 12 , tumour necrosis factor $\alpha$, interleukin $1 \beta$, interleukin 1 receptor antagonist, granulo cyte colony-stimulating factor, and chemokine (C-X-C motif) ligand 10 were also measured, with analysis done by Charles River Laboratories Preclinical Services (Montreal, QC, Canada). 
PCSK9 and LDL cholesterol samples for screening and on-study assessments (obtained after an overnight fast) were analysed at accredited centralised laboratories: plasma PCSK9 was measured by ELISA (Charles River Laboratories, Montreal, QC, Canada) and LDL choles terol, total cholesterol, and HDL cholesterol were measured directly by $\beta$-quantification (Medpace Reference Laboratories, Cincinnati, OH, USA and Leuven, Belgium).

For exploratory purposes, changes in very low-density lipoprotein (VLDL) cholesterol, ApoB, and lipo pro tein A were also examined in the higher-dose groups $(\geq) \cdot 150 \mathrm{mg} / \mathrm{kg}$; ApoB and lipoprotein A analyses were not prespecified).

The primary endpoint was safety and tolerability. Safety was assessed by the frequency and severity of adverse events, including the protocol-defined stopping rules. The numbers and proportions of participants with any treatment-emergent adverse event, any treatmentemergent adverse event assessed by the treating investigator as related to study drug, and any serious treatment-emergent adverse event were summarised by dose. Adverse events were coded with the Medical Dictionary for Regulatory Activities (version 14.1).

Secondary endpoints were the pharma cokinetics of ALN-PCS and its pharmacodynamic effects on fasting plasma PCSK9 and serum LDL cholesterol. Changes in fasting HDL cholesterol, total cholesterol, and VLDL cholesterol were also measured (post-hoc analyses). In the higher-dose groups, lipoprotein A and ApoB were also examined post hoc.

\section{Statistical analysis}

All statistical analyses were done with SAS (version 9.2 or later) or R (version 2.13 .2 or later). Ratios of PCSK9 protein and LDL cholesterol, both relative to baseline and relative to baseline and placebo (individual reduc tions scaled to placebo-group mean per day), were log-transformed before analysis. For all traits for which pairwise comparisons of individual dose groups at particu lar timepoints were to be done, repeated-measures ANCOVA first established signifi cant time, dose, and time-by-dose interaction effects. The ANCOVA models included dose group, time, and individual participant as factors; trait baseline as a covariate; and dose by time as the sole interaction term.

Significance of pairwise comparisons was then assessed by $t$ tests (with Bonferroni correction for multiple com parisons), and ratios were back-transformed to percen tages for presentation. Per centage change versus placebo (the difference between dose group and placebo group percentage reduction relative to their respective baselines) was estimated from a similar ANCOVA model that used percentage without trans formation, and $\mathrm{p}$ values were determined in the same way as for pairwise com parisons of dose groups.

Single-factor ANOVAs with Tukey's post-hoc tests were used to determine the significance of changes in total cholesterol and HDL cholesterol. ANCOVA models examined PCSK9 and LDL cholesterol reduction at per-individual nadir (days 1-28). The models included baseline PCSK9 or LDL cholesterol as covariates, and dose group as a factor. Least-square means and 95\% CIs were estimated from the ANCOVA models. Tukey's tests (with Bonferroni correction) assessed the significance of pairwise comparisons of individual dose groups' least-square means versus placebo. 
The trial is registered with ClinicalTrials.gov, number NCT01437059.

\section{Role of the funding source}

The sponsor contributed to the study at all stages, including trial design, sample testing, statistical analysis, data interpretation, and writing of this report. Prometrika (Cambridge, MA, USA) was used as a contract organisation for some of the statistical analysis. All authors had access to the primary data. KF and AS had primary responsibility for the decision to submit for publication.

\section{Results}

32 eligible participants were recruited between Sept 21, 2011, and Sept 11, 2012. 24 were randomly assigned to receive ALN-PCS and eight to receive placebo (figure 2). One participant was excluded after random assignment because their lipid concentrations proximal to dosing were outside of those defined by the inclusion criteria. Table 1 lists the baseline characteristics of all participants who received study treatment and therefore were included in the analyses.

Overall, ALN-PCS was safe and well tolerated. No drug-related serious adverse events occurred in any of the participants who received ALN-PCS. One serious adverse event occurred during the study in a participant given the $0.045 \mathrm{mg} / \mathrm{kg}$ dose, who was diagnosed with bilateral pulmonary emboli and a deep vein thrombosis on day 3 of the study. This event was at one of the lower doses in the study and was determined by the investigator and safety review com mittee to be unrelated to study drug, in view of the individual's medical history of a similar episode of chest pain 3 months previously, and the presence of symptoms of thigh pain before dosing (both of which were un disclosed by the participant during the study screening assessments), as well as ultrasound evidence of collateral circulation pathways circumventing the deep vein throm bosis of the leg, which suggested a chronic disease process. Similar neutral lipid nano-particles have been given at higher doses and chronically in patients with no evidence of thrombosis. ${ }^{35,36}$ All treatment-emergent adverse events were mild to moderate in severity, with similar proportions affected in the ALN-PCS and placebo groups (19 [79\%] vs seven [88\%]; table 2). A mild, macular, erythematous rash occurred with equal frequency in participants given ALN-PCS and those given placebo. The rash was transient, occurring on the first day after treatment, and was asymptomatic without pain or pruritus. We noted no clinically significant dose-dependent changes in any laboratory indices, including liver func tion tests, creatine phosphokinase, Creactive pro tein, and haematological measures. No clinically sig nifi cant dose-dependent changes occurred in concen trations of the nine cytokines measured. Finally, we noted no clinically signifi cant safety findings during the 6 month follow-up period.

The plasma pharmacokinetic profiles of the ALN-PCS siRNA showed rapid distribution in the plasma, with peak concentration occurring at the end of infusion or shortly thereafter. Peak concentration and area under the curve ( 0 to last measurement) increased in a roughly dose-proportional manner across the dose range tested (appendix $\mathrm{p} 7$ ). 
Single-dose administration of ALN-PCS resulted in a rapid and dose-dependent reduction in plasma PCSK9 protein (figures 3A, 3B). The duration of PCSK9 lowering was also dosedependent, with higher doses resulting in more prolonged reduction (figures $3 \mathrm{~A}, 3 \mathrm{~B}$ ). At the highest dose of $0.400 \mathrm{mg} / \mathrm{kg}$, PCSK9 fell by $70 \%$ from baseline relative to placebo on day 3 post-dose ( $<<0 \cdot 0001$; figure $3 \mathrm{~A}$, table 3 ), with an individual maximum reduction of $84 \%$. Similar to the arithmetic mean data, the least-square mean individual maximum reduction in plasma PCSK9 protein at the $0.400 \mathrm{mg} / \mathrm{kg}$ dose was $68 \%$ (figure 3B).

Plasma PCSK9 increased significantly in the placebo group, beginning on day 1, peaking on day 2 , and returning to baseline values on day 7 (figure $3 \mathrm{C}$, table 3 ). This increase in the placebo group was attributed to the premedication given to all participants, and was clearly overcome in a dose-dependent fashion by ALN-PCS treatment (figure 3A, 3C, table 3, appendix p 8). The effect of ALN-PCS was sustained well beyond day 7, when the effects of the premedication were absent (figure 3C, table 3, figure 4A). To confirm that the effect of ALN-PCS was specific for PCSK9, we also measured changes in plasma transthyretin, a liver-secreted protein that was not expected to change with liver silencing of PCSK9. We noted no significant change in plasma transthyretin concentration after treatment with ALNPCS at $0.400 \mathrm{mg} / \mathrm{kg}$ (appendix p 8), suggesting that the effect of ALN-PCS on plasma PCSK9 concentration was target specific. Baseline values of PCSK9 can vary by a factor of more than 100 across the population; ${ }^{37}$ however, since RNAi drugs function by a catalytic mechanism and ALN-PCS blocks PCSK9 synthesis, we predicted that the reductions in plasma PCSK9 would be independent of the baseline PCSK9 concentrations for each individual participant, and this prediction was confirmed by our results (figure 3D).

As expected from the significant lowering of plasma PCSK9, single-dose administration of ALN-PCS also resulted in a rapid and dose-dependent reduction in serum LDL cholesterol (figures 4A, 4B). The duration of LDL cholesterol lowering was also dose-dependent, with higher doses resulting in more prolonged reduction (figures 4A, 4B). Administration of the highest dose $(0.400 \mathrm{mg} / \mathrm{kg})$ resulted in a mean $40 \%$ reduction $(\mathrm{p}<0.0001)$ from baseline relative to placebo (figure $4 \mathrm{~A}$, table 3 ), with an individual maximum reduction of $57 \%$. Similar to the arithmetic mean data, the least-square mean individual maximum redwuction in LDL cholesterol was $42 \%$ ( $\mathrm{p}=0.0021$; figure 4B). In addition to the effects of premedication on PCSK9, ALN-PCS treatment also had an apparent effect on LDL cholesterol at early timepoints, with a reduction noted on day 2 that reached a nadir at day 4 and recovered to baseline by day 7 (figure $4 \mathrm{C}$, table 3 , appendix $\mathrm{p} 8$ ). The effects of ALNPCS on LDL cholesterol were clearly sustained well past the time at which LDL cholesterol concentrations in participants given placebo had recovered to baseline (figure 4C, table 3 ). The number of participants who reached an LDL cholesterol concentration less than $2.6 \mathrm{mmol} / \mathrm{L}$ increased with increasing dose (figure 4D). The appendix (p 5) lists the actual values of LDL cholesterol for individual participants. We also noted changes in total cholesterol, but not in HDL cholesterol (appendix pp 1-4).

\section{Discussion}

ALN-PCS was well tolerated, with similar proportions of mild to moderate treatmentemergent adverse events occurring in the treatment and placebo groups. Although some 
participants developed a rash, it was mild, resolved spontaneously, and occurred with equal frequency in the in placebo and ALN-PCS-treated participants. The rash was identical in appearance and nature in both groups, and was probably a result of the premedications given to all participants, which can cause skin flushing (due to vasodilatation) or a skin rash. Ascertainment bias might have led to an increase in the reported frequency of rash in both groups as the dose of ALN-PCS increased, giving the impression of a dose-responsive effect; the higher-dose cohorts ( $\searrow 0 \cdot 150 \mathrm{mg} / \mathrm{kg}$ ) were treated exclusively at one site (Covance Clinical Research Unit), and this site reported a higher frequency of rash than the second site in both the treatment and placebo groups. We noted no clinically significant changes in liver function tests, troponin, or inflammatory markers (ie, cytokines and C-reactive protein). Despite these encouraging safety findings, additional, larger, multidose studies are needed to confirm the safety and tolerability of ALN-PCS.

Although this was a small phase 1 study that was not powered to detect changes in PCSK9 or LDL cholesterol, we did note a significant lowering of plasma PCSK9 and serum LDL cholesterol in the higher-dose groups. These effects were rapid and durable after a single dose, potentially supporting a dosing schedule of once or twice per month in future multidose studies (panel).

The data in human beings were well predicted from preclinical studies in non-human primates (appendix p 6), and our results suggest that dosing translated from pri mates to human beings on a roughly $1: 1 \mathrm{mg} / \mathrm{kg}$ basis. Because this study was the first time ALN-PCS was given to human beings, participants were given premedication to prevent any potential infusion-related reactions that can occur with lipid-formulated drugs. The premedication regi men resulted in a sub stantial increase in serum PCSK9 concentrations and a small reduction in serum LDL cholesterol in the placebo group at early timepoints, with these measures return ing to baseline by day 7 . Although this effect was fully accounted for in our statistical analysis, we suggest a cautious approach to interpreting the absolute size of the changes in PCSK9 and LDL cholesterol con centrations seen at the early time points of the study.

Although working by very different mechanisms (figure 1), antibodies and ALN-PCS both seem to be effective at blocking PCSK9 function and thereby inducing a reduction in LDL and total cholesterol. ALN-PCS was given intravenously in this study and its effect on LDL cholesterol was less than that reported for the highest dose groups of anti-PCSK9 antibodies, with AMG 145 lowering LDL cholesterol by up to $67 \%$ at the highest dose when given to healthy volunteers. ${ }^{23,24}$ Statins such as atorvastatin range in efficacy with respect to the lowering of LDL cholesterol from $36 \%$ to $53 \%$, dependent on the patient population and the dose given. ${ }^{38}$ A maximum tolerated dose of ALN-PCS was not reached in this study and premedication resulted in artificially high values of PCSK9 and reduced LDL cholesterol at early timepoints. Therefore, future studies that use higher doses of ALN-PCS without premedication and in patients on statin treatment are warranted.

Studies with anti-PCSK9 antibodies in healthy individuals have shown increased HDL cholesterol and decreased lipoprotein A. ${ }^{23,24}$ In this study we noted no significant changes in either in response to ALN-PCS (appendix pp 1-4), although the number of participants assessed at efficacious doses was small. 
The seminal discovery of RNAi as a conserved bio logical mechanism ${ }^{39}$ took place in 1998 and was awarded the Nobel Prize in Physiology or Medicine in 2006. This study is the first definitive clinical proof of concept in human beings of an RNAi drug being used to lower a liver-derived protein (PCSK9), thereby resulting in favourable changes in a clinically validated endpoint (LDL cholesterol). These results pave the way for add itional siRNA treatments targeted at liver-expressed genes implicated in human disease.

\section{Supplementary Material}

Refer to Web version on PubMed Central for supplementary material.

\section{Acknowledgments}

The study was sponsored by Alnylam Pharmaceuticals (Cambridge, MA, USA). Some sample testing and one author $(\mathrm{JH})$ were supported by NIH grant number HL020948. We thank the Alnylam CMC, quality, chemistry, project management, and regulatory PCS core team members Lubomir Nechev, David Watkins, David Konys, Lauri Binne, Matthias Kretschmer, Jason Costigan, Kimberly Smith, and Tim Buck for their contributions to the supply and release of ALN-PCS and the writing, editing, and filing of the clinical trial authorisation document. We also thank the Alnylam formulations team led by Akin Akinc and Martin Maier, the Alnylam screening and lead finding group led by Rachel Meyers, and the Alnylam chemistry team led by Muthiah Manoharan. Finally, we thank Maryellen Livingston for her help in preparing the report, and John Maraganore for his critical appraisal of the report before submission.

\section{References}

1. Grundy SM, Cleeman JI, Merz CN, et al. Implications of recent clinical trials for the National Cholesterol Education Program Adult Treatment Panel III guidelines. J Am Coll Cardiol. 2004; 44:720-32. [PubMed: 15358046]

2. Delahoy PJ, Magliano DJ, Webb K, Grobler M, Liew D. The relationship between reduction in lowdensity lipoprotein cholesterol by statins and reduction in risk of cardiovascular outcomes: an updated meta-analysis. Clin Ther. 2009; 31:236-44. [PubMed: 19302897]

3. Sniderman A, Thanassoulis G, Couture P, Williams K, Alam A, Furberg CD. Is lower and lower better and better? A re-evaluation of the evidence from the Cholesterol Treatment Trialists' Collaboration meta-analysis for low-density lipoprotein lowering. J Clin Lipidol. 2012; 6:303-09. [PubMed: 22836065]

4. Davidson MH, Maki KC, Pearson TA, et al. Results of the National Cholesterol Education (NCEP) Program Evaluation ProjecT Utilizing Novel E-Technology (NEPTUNE) II survey and implications for treatment under the recent NCEP Writing Group recommendations. Am J Cardiol. 2005; 96:556-63. [PubMed: 16098311]

5. Foley KA, Simpson RJ Jr, Crouse JR 3rd, Weiss TW, Markson LE, Alexander CM. Effectiveness of statin titration on low-density lipoprotein cholesterol goal attainment in patients at high risk of atherogenic events. Am J Cardiol. 2003; 92:79-81. [PubMed: 12842255]

6. Cholesterol Treatment Trialists' (CTT) Collaborators. Efficacy and safety of cholesterol-lowering treatment: prospective meta-analysis of data from 90056 participants in 14 randomised trials of statins. Lancet. 2005; 366:1267-78. [PubMed: 16214597]

7. Cholesterol Treatment Trialists' (CTT) Collaborators. Efficacy of cholesterol-lowering therapy in 18686 people with diabetes in 14 randomised trials of statins: a meta-analysis. Lancet. 2008; 371:117-25. [PubMed: 18191683]

8. Genest J, McPherson R, Frohlich J, et al. Canadian Cardiovascular Society/Canadian guidelines for the diagnosis and treatment of dyslipidemia and prevention of cardiovascular disease in the adult2009 recommendations. Can J Cardiol 2009. 2009; 25:567-79.

9. Foody JM, Sajjan SG, Hu XH, et al. Loss of early gains in low-density lipoprotein cholesterol goal attainment among high-risk patients. J Clin Lipidol. 2010; 4:126-32. [PubMed: 21122640] 
10. Thomas JE, Tershakovec AM, Jones-Burton C, Sayeed RA, Foody JM. Lipid lowering for secondary prevention of cardiovascular disease in older adults. Drugs Aging. 2010; 27:959-72. [PubMed: 21087066]

11. Abifadel M, Varret M, Rabes JP, et al. Mutations in PCSK9 cause autosomal dominant hypercholesterolemia. Nat Genet. 2003; 34:154-56. [PubMed: 12730697]

12. Horton JD, Cohen JC, Hobbs HH. Molecular biology of PCSK9: its role in LDL metabolism. Trends Biochem Sci. 2007; 32:71-77. [PubMed: 17215125]

13. Poirier S, Mayer G, Poupon V, et al. Dissection of the endogenous cellular pathways of PCSK9induced low density lipoprotein receptor degradation: evidence for an intracellular route. J Biol Chem. 2009; 284:28856-64. [PubMed: 19635789]

14. Cohen JC, Boerwinkle E, Mosley TH Jr, Hobbs HH. Sequence variations in PCSK9, low LDL, and protection against coronary heart disease. N Engl J Med. 2006; 354:1264-72. [PubMed: 16554528]

15. Horton JD, Cohen JC, Hobbs HH. PCSK9: a convertase that coordinates LDL catabolism. J Lipid Res. 2009; 50(suppl):S172-77. [PubMed: 19020338]

16. Zhao Z, Tuakli-Wosornu Y, Lagace TA, et al. Molecular characterization of loss-of-function mutations in PCSK9 and identification of a compound heterozygote. Am J Hum Genet. 2006; 79:514-23. [PubMed: 16909389]

17. Hooper AJ, Marais AD, Tanyanyiwa DM, Burnett JR. The C679X mutation in PCSK9 is present and lowers blood cholesterol in a Southern African population. Atherosclerosis. 2007; 193:44548. [PubMed: 16989838]

18. Rashid S, Curtis DE, Garuti R, et al. Decreased plasma cholesterol and hypersensitivity to statins in mice lacking Pcsk9. Proc Natl Acad Sci USA. 2005; 102:5374-79. [PubMed: 15805190]

19. Careskey HE, Davis RA, Alborn WE, Troutt JS, Cao G, Konrad RJ. Atorvastatin increases human serum levels of proprotein convertase subtilisin/kexin type 9. J Lipid Res. 2008; 49:394-98. [PubMed: 18033751]

20. Davignon J, Dubuc G. Statins and ezetimibe modulate plasma proprotein convertase subtilisin kexin-9 (PCSK9) levels. Trans Am Clin Climatol Assoc. 2009; 120:163-73. [PubMed: 19768174]

21. Costet P, Hoffmann MM, Cariou B, et al. Plasma PCSK9 is increased by fenofibrate and atorvastatin in a non-additive fashion in diabetic patients. Atherosclerosis. 2010; 212:246-51. [PubMed: 20619837]

22. Welder G, Zineh I, Pacanowski MA, Troutt JS, Cao G, Konrad RJ. High-dose atorvastatin causes a rapid sustained increase in human serum PCSK9 and disrupts its correlation with LDL cholesterol. J Lipid Res. 2010; 51:2714-21. [PubMed: 20525997]

23. Stein EA, Mellis S, Yancopoulos GD, et al. Effect of a monoclonal antibody to PCSK9 on LDL cholesterol. N Engl J Med. 2012; 366:1108-18. [PubMed: 22435370]

24. Dias CS, Shaywitz AJ, Wasserman SM, et al. Effects of AMG 145 on low-density lipoprotein cholesterol levels: results from 2 randomized, double-blind, placebo-controlled, ascending-dose phase 1 studies in healthy volunteers and hypercholesterolemic subjects on statins. J Am Coll Cardiol. 2012; 60:1888-98. [PubMed: 23083772]

25. Raal F, Scott R, Somaratne R, et al. Low-density lipoprotein cholesterol-lowering effects of AMG 145 , a monoclonal antibody to proprotein convertase subtilisin/kexin type 9 serine protease in patients with heterozygous familial hypercholesterolemia: the Reduction of LDL-C with PCSK9 Inhibition in Heterozygous Familial Hypercholesterolemia Disorder (RUTHERFORD) randomized trial. Circulation. 2012; 126:2408-17. [PubMed: 23129602]

26. Sullivan D, Olsson AG, Scott R, et al. Effect of a monoclonal antibody to PCSK9 on low-density lipoprotein cholesterol levels in statin-intolerant patients: the GAUSS randomized trial. JAMA. 2012; 308:2497-506. [PubMed: 23128163]

27. Roth EM, McKenney JM, Hanotin C, Asset G, Stein EA. Atorvastatin with or without an antibody to PCSK9 in primary hypercholesterolemia. N Engl J Med. 2012; 367:1891-900. [PubMed: 23113833]

28. Banerjee Y, Shah K, Al-Rasadi K. Effect of a monoclonal antibody to PCSK9 on LDL cholesterol. N Engl J Med. 2012; 366:2425-26. [PubMed: 22716984] 
29. Milazzo L, Antinori S. Effect of a monoclonal antibody to PCSK9 on LDL cholesterol. N Engl J Med. 2012; 366:2425. [PubMed: 22716983]

30. Hooper AJ, Burnett JR. Anti-PCSK9 therapies for the treatment of hypercholesterolemia. Expert Opin Biol Ther. 2013; 13:429-35. [PubMed: 23240807]

31. Meister G, Tuschl T. Mechanisms of gene silencing by double-stranded RNA. Nature. 2004; 431:343-49. [PubMed: 15372041]

32. Vaishnaw AK, Gollob J, Gamba-Vitalo C, et al. A status report on RNAi therapeutics. Silence. 2010; 1:14. [PubMed: 20615220]

33. Frank-Kamenetsky M, Grefhorst A, Anderson NN, et al. Therapeutic RNAi targeting PCSK9 acutely lowers plasma cholesterol in rodents and LDL cholesterol in nonhuman primates. Proc Natl Acad Sci USA. 2008; 105:11915-20. [PubMed: 18695239]

34. Jayaraman M, Ansell SM, Mui BL, et al. Maximizing the potency of siRNA lipid nanoparticles for hepatic gene silencing in vivo. Angew Chem Int Ed Engl. 2012; 51:8529-33. [PubMed: 22782619]

35. Tabernero J, Shapiro GI, LoRusso PM, et al. First-in-humans trial of an RNA interference therapeutic targeting VEGF and KSP in cancer patients with liver involvement. Cancer Discov. 2013; 3:406-17. [PubMed: 23358650]

36. Barros SA, Gollob JA. Safety profile of RNAi nanomedicines. Adv Drug Deliv Rev. 2012; 64:1730-37. [PubMed: 22732527]

37. Lakoski SG, Lagace TA, Cohen JC, Horton JD, Hobbs HH. Genetic and metabolic determinants of plasma PCSK9 levels. J Clin Endocrinol Metab. 2009; 94:2537-43. [PubMed: 19351729]

38. Adams SP, Tsang M, Wright JM. Lipid lowering efficacy of atorvastatin. Cochrane Database Syst Rev. 2012; 12:CD008226. [PubMed: 23235655]

39. Fire A, Xu S, Montgomery MK, Kostas SA, Driver SE, Mello CC. Potent and specific genetic interference by double-stranded RNA in Caenorhabditis elegans. Nature. 1998; 391:806-11. [PubMed: 9486653] 


\section{Panel: Research in context}

Systematic review

In 2006, Cohen and colleagues, ${ }^{14}$ first reported that heterozygous loss-of-function mutations in PCSK9 resulted in individuals having lower than average LDL cholesterol and a greatly reduced risk of cardiovascular disease. Since that report investigators have focused on the development of drugs to lower the activity of PCSK9 and thereby increase LDL receptor concentrations and decrease plasma cholesterol. We searched PubMed for all articles published in English up to June 12, 2013, that contained the MeSH terms "PCSK9" or "proprotein convertase subtilisin kexin" in the abstract or title. We identified several publications that described PCSK9 inhibitors that had been assessed in clinical trials. $^{23-29}$ These reports showed that inhibiting PCSK9 by blocking its activity with an antibody lowers LDL cholesterol in healthy volunteers and in patients with hyperlipidaemia with or without concomitant treatment with statin treatment. We found no reports of RNA interference (RNAi)-based synthesis inhibitors of PCSK9.

Interpretation

ALN-PCS, an RNAi drug designed to inhibit the synthesis of the PCSK 9 transcript and thus reduce plasma PCSK9, was well tolerated and significantly lowered PCSK9 and LDL cholesterol after a single intravenous dose in healthy volunteers with raised cholesterol. Our study is the first systemic trial of an RNAi drug to report an effect on a clinically validated endpoint (LDL cholesterol). Future trials are needed to fully assess the benefit and long-term safety of ALN-PCS in various patient populations, including those on statins, those who are statin intolerant, and those with high baseline PCSK9 due to defects in LDL receptor function. 


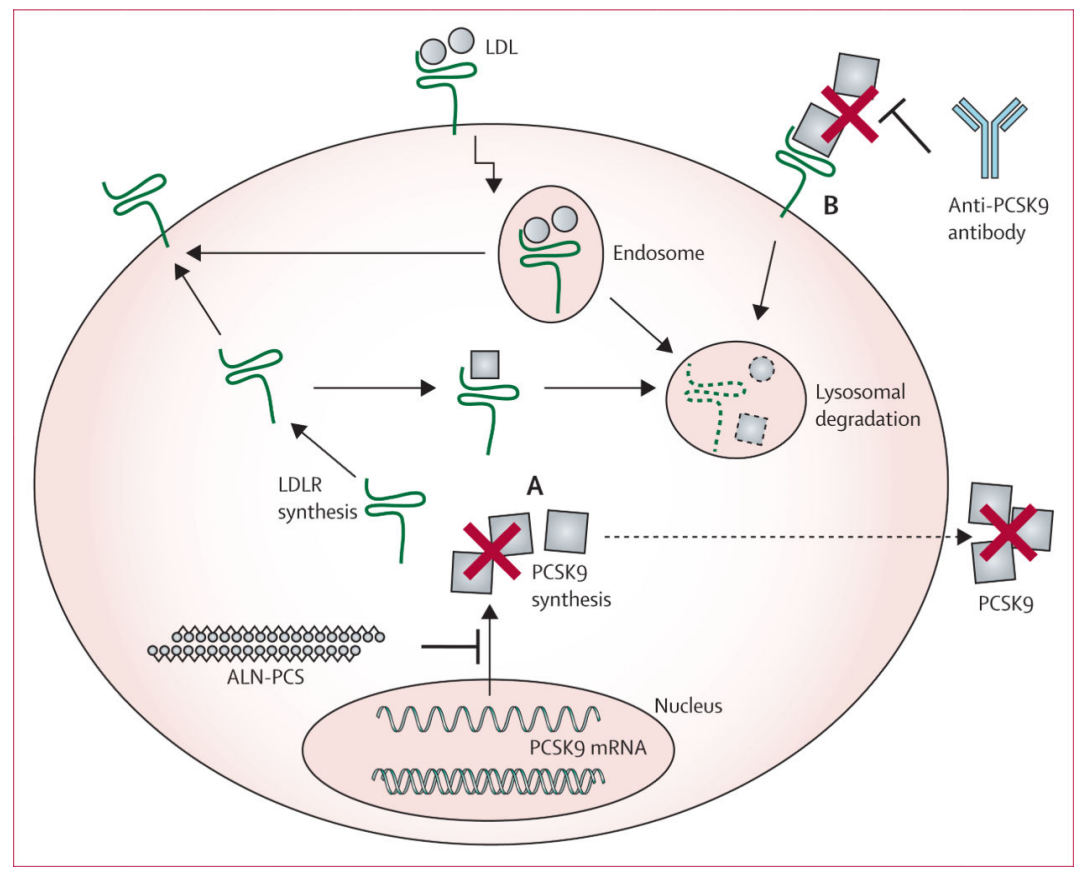

Figure 1. PCSK9 pathway and RNA interference synthesis-inhibitor approach

PCSK9 has a role in both intracellular and extracellular degradation of the LDL receptor (LDLR). PCSK9 synthesis inhibitors such as ALN-PCS inhibit PCSK9 synthesis (A) and therefore both intracellular and extracellular functions, whereas PCSK9 blockers (such as anti-PCSK9 antibodies) inhibit only extracellular function (B). mRNA=messenger RNA. 


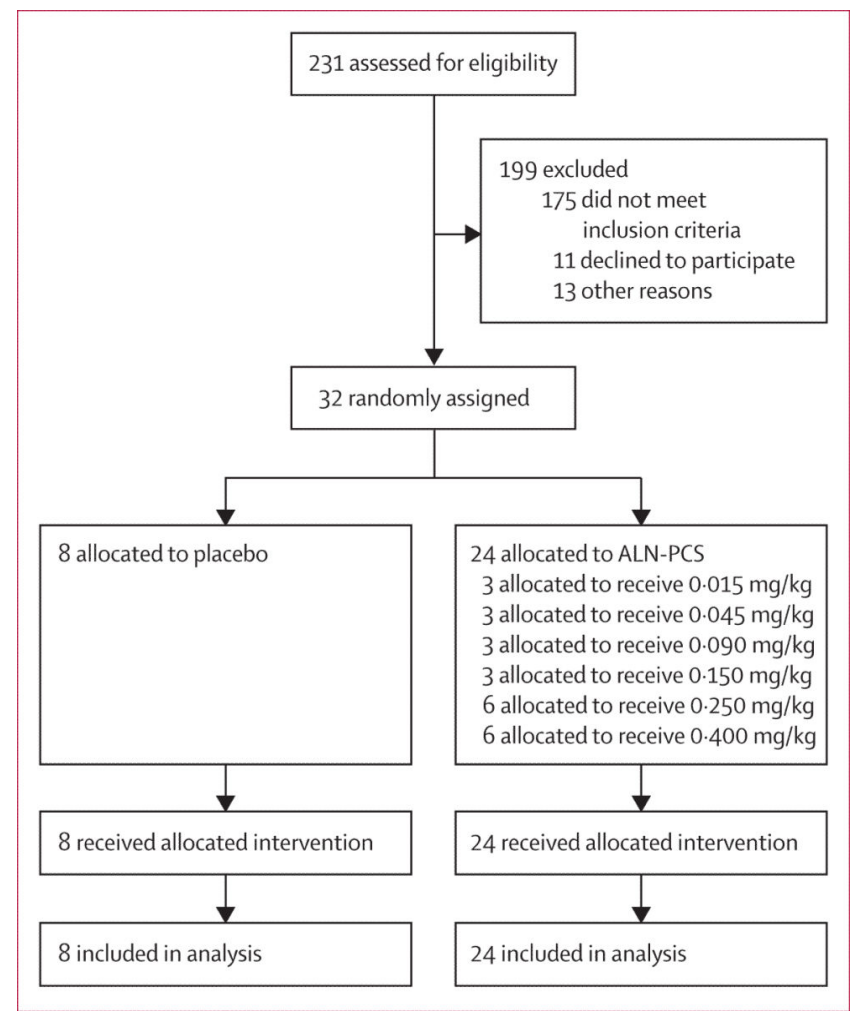

Figure 2.

Trial profile 


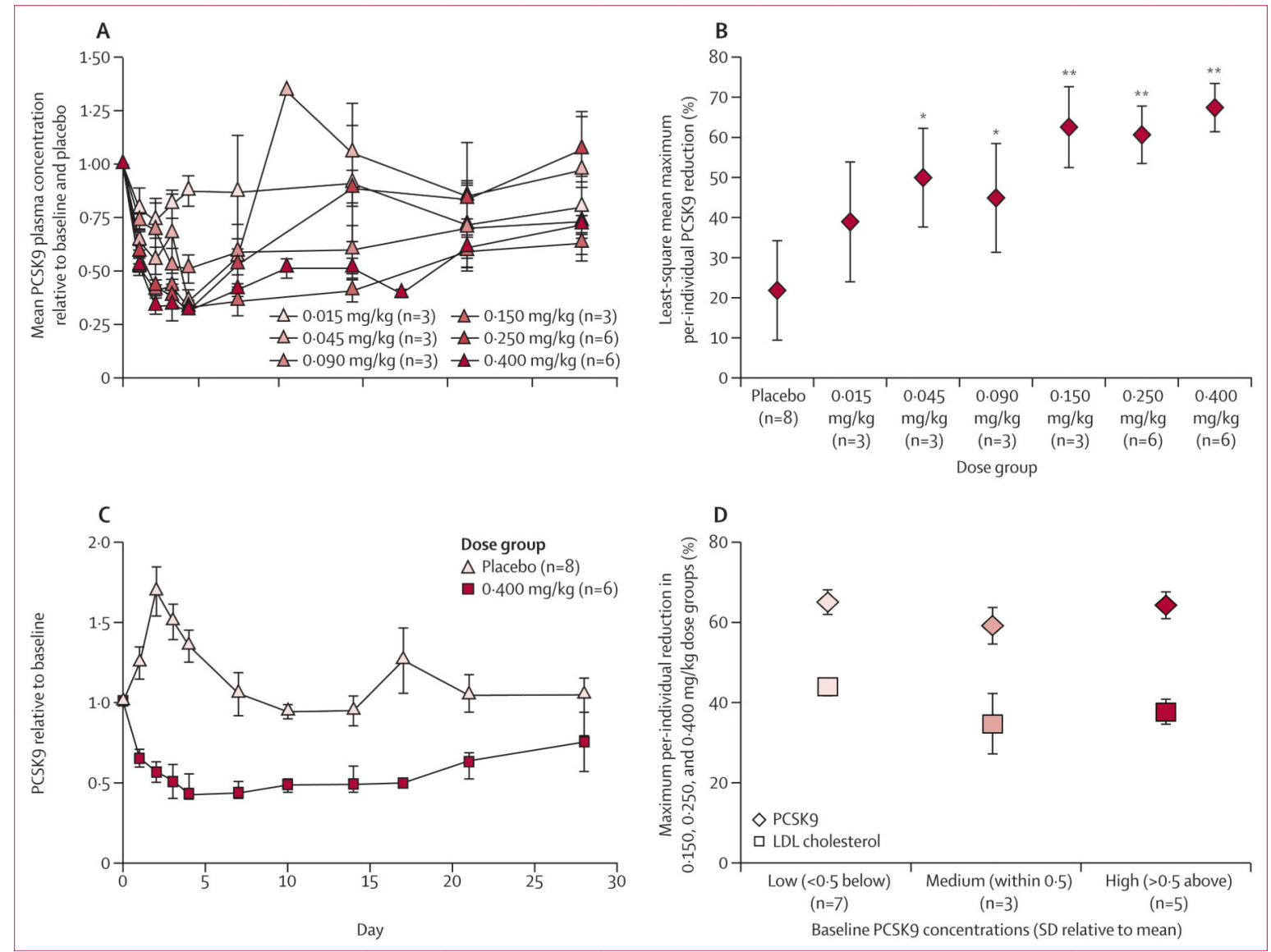

Figure 3. Effect of ALN-PCS treatment on plasma PCSK9 concentration

(A) Mean plasma PCSK9 concentrations relative to baseline and placebo. Error bars are SEs. PCSK9 concentrations were normalised per-individual to baseline and then per-day to placebo group means. For $0 \cdot 045 \mathrm{mg} / \mathrm{kg}$ group at day 10 , data from only one of three participants were available; for days 10 and 17, blood samples were only available for the $0.250 \mathrm{mg} / \mathrm{kg}$ and $0.400 \mathrm{mg} / \mathrm{kg}$ dose groups and their affiliated placebo participants, so values at these days are derived from or normalised to data from two of eight placebo participants. (B) Maximum plasma PCSK9 percentage reduction after treatment with ALNPCS. Maximum reductions were determined per individual, at lowest PCSK9 value from days 1-28. Data are least-square means and error bars are 95\% CIs, determined from analysis of covariance with baseline PCSK9 as covariate. ${ }^{*} \mathrm{p}<0.05$; **p<0.001 (Tukey's post-hoc tests $v s$ placebo). Least-square mean PCSK9 reductions per dose group were estimated via a linear model with dose group as a factor and baseline PCSK9 as a covariate. The overall model was significant $\left(F=8 \cdot 821 ; \mathrm{p}=2 \cdot 307 \times 10^{5}\right)$. (C) Mean plasma PCSK9 concentrations relative to baseline. Error bars are SEs. (D) Mean maximum percentage reductions in PCSK9 and LDL cholesterol in participants given $0 \cdot 150,0 \cdot 250$, and $0 \cdot 400$ $\mathrm{mg} / \mathrm{kg}$ ALN-PCS, grouped by low, intermediate, and high baseline PCSK 9 concentrations $(<0 \cdot 5$, within $0 \cdot 5$, and $>0 \cdot 5 \mathrm{SDs}$ from the mean, respectively). Error bars are SEs. 


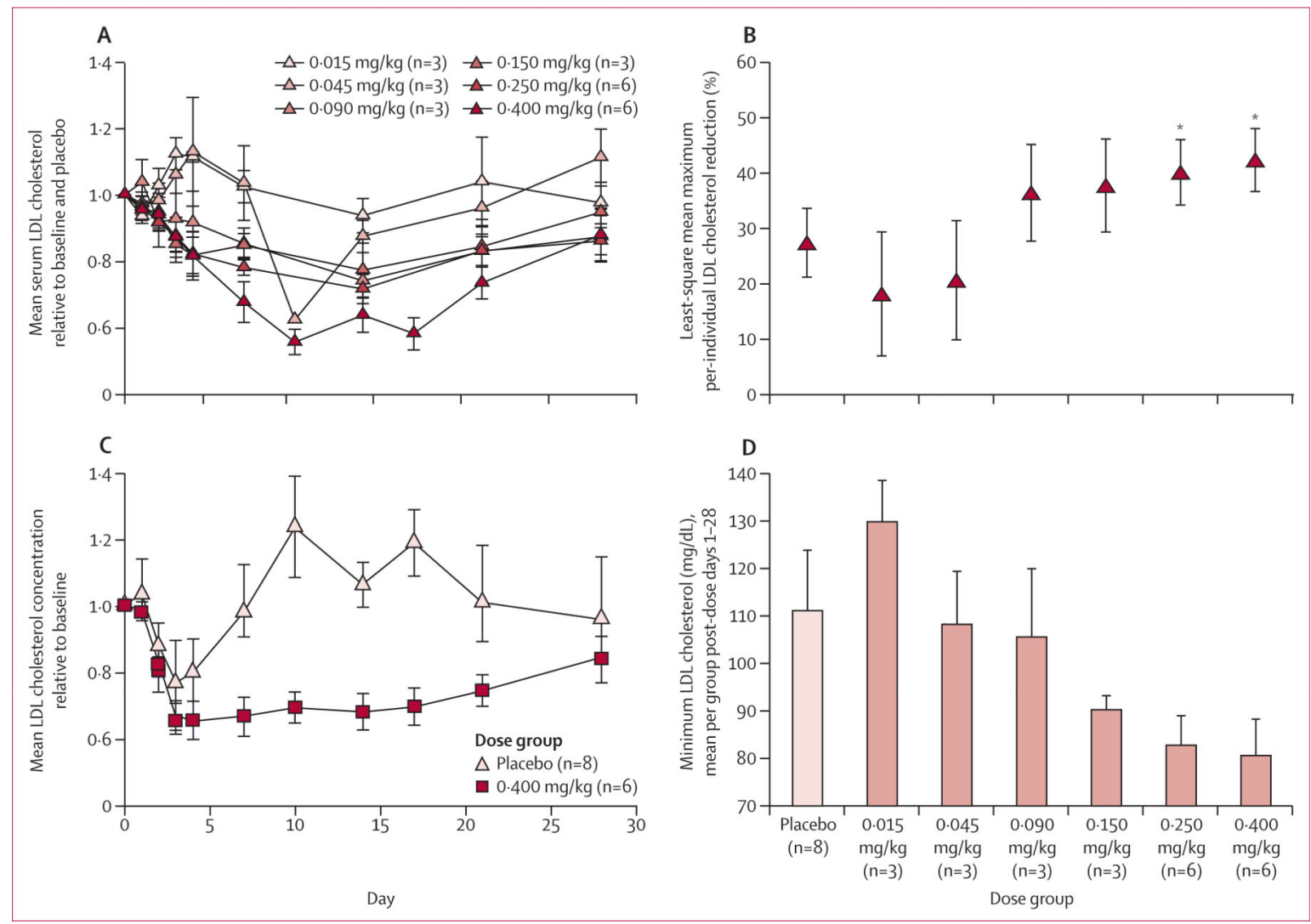

Figure 4. Effect of ALN-PCS treatment on serum LDL cholesterol

(A) Mean serum LDL cholesterol concentrations relative to baseline and placebo. Error bars are SEs. LDL cholesterol concentrations were normalised per-individual to baseline and then per-day to placebo group means. For $0.045 \mathrm{mg} / \mathrm{kg}$ group at day 10 , data from only one of three participants were available; for days 10 and 17, blood samples were only available for the $0.250 \mathrm{mg} / \mathrm{kg}$ and $0.400 \mathrm{mg} / \mathrm{kg}$ dose groups and their affiliated placebo participants, so values at these days are derived from or normalised to data from two of eight placebo participants. (B) Maximum serum LDL cholesterol percentage reductions after treatment with ALN-PCS. Maximum reductions were determined per individual, at nadir LDL cholesterol value from days 1-28. Data are least-square means and error bars are 95\% CIs, determined from analysis of covariance with baseline LDL cholesterol as covariate. * $\mathrm{p}<0 \cdot 01$ (Tukey's post hoc test $v s$ placebo). Least-square mean LDL cholesterol reductions per dose group were estimated via a linear model with dose group as a factor and baseline LDL cholesterol as a covariate. The overall model was significant $(F=4.888 ; \mathrm{p}=0.0015)$. (C) Mean serum LDL cholesterol concentrations relative to baseline and placebo. Error bars are SEs. For days 10 and 17, blood samples were only available for the $0.250 \mathrm{mg} / \mathrm{kg}$ and 0.400 $\mathrm{mg} / \mathrm{kg}$ dose groups and their afliliated placebo participants, so values at these days are derived from or normalised to data from two of eight placebo participants. (D) Mean serum LDL cholesterol concentrations at per-individual nadir from days 1-28. Error bars are SEs. 
Table 1

Baseline characteristics

\begin{tabular}{|lll|}
\hline & ALN-PCS group $(\mathbf{n}=24)$ & Placebo group $(\mathbf{n = 8})$ \\
\hline Median age (years) & $51 \cdot 0(25 \cdot 0-60 \cdot 0)$ & $41 \cdot 5(33 \cdot 0-61 \cdot 0)$ \\
Sex & & \\
$\quad$ Male & 22 & 8 \\
Female & 2 & 0 \\
Mean body-mass index & $28 \cdot 7(23 \cdot 4-33 \cdot 9)$ & $28 \cdot 9(24 \cdot 7-33 \cdot 4)$ \\
Mean baseline PCSK9 (ng/mL) & $1014 \cdot 7(453 \cdot 2-1528 \cdot 7)$ & $1067 \cdot 0(788 \cdot 9-1355 \cdot 1)$ \\
Mean baseline LDL cholesterol $(\mathrm{mmol} / \mathrm{L})$ & $3 \cdot 7(2 \cdot 9-4 \cdot 8)$ & $3 \cdot 9(3 \cdot 0-6 \cdot 0)$ \\
Mean baseline HDL cholesterol (mmol/L) & $1 \cdot 3(0 \cdot 8-2 \cdot 3)$ & $1 \cdot 2(0 \cdot 8-1 \cdot 6)$ \\
Mean baseline total cholesterol $(\mathrm{mmol} / \mathrm{L})$ & $5 \cdot 7(4 \cdot 3-6 \cdot 6)$ & $5 \cdot 7(4 \cdot 5-8 \cdot 1)$ \\
\hline
\end{tabular}

Data are $\mathrm{n}$, median (range), or mean (range). PCSK9 plasma values are based on ELISA. Cholesterol values are based on $\beta$-quantification measurements. 
Fitzgerald et al.

Page 19

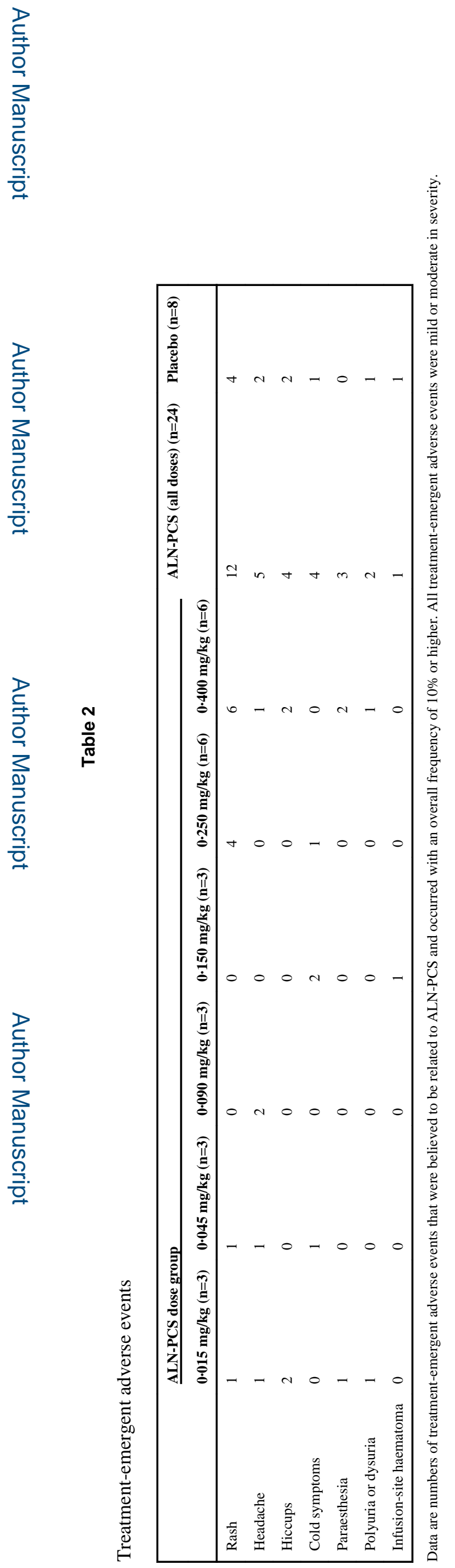

Lancet. Author manuscript; available in PMC 2015 April 07. 


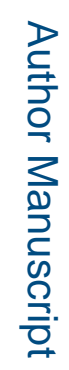

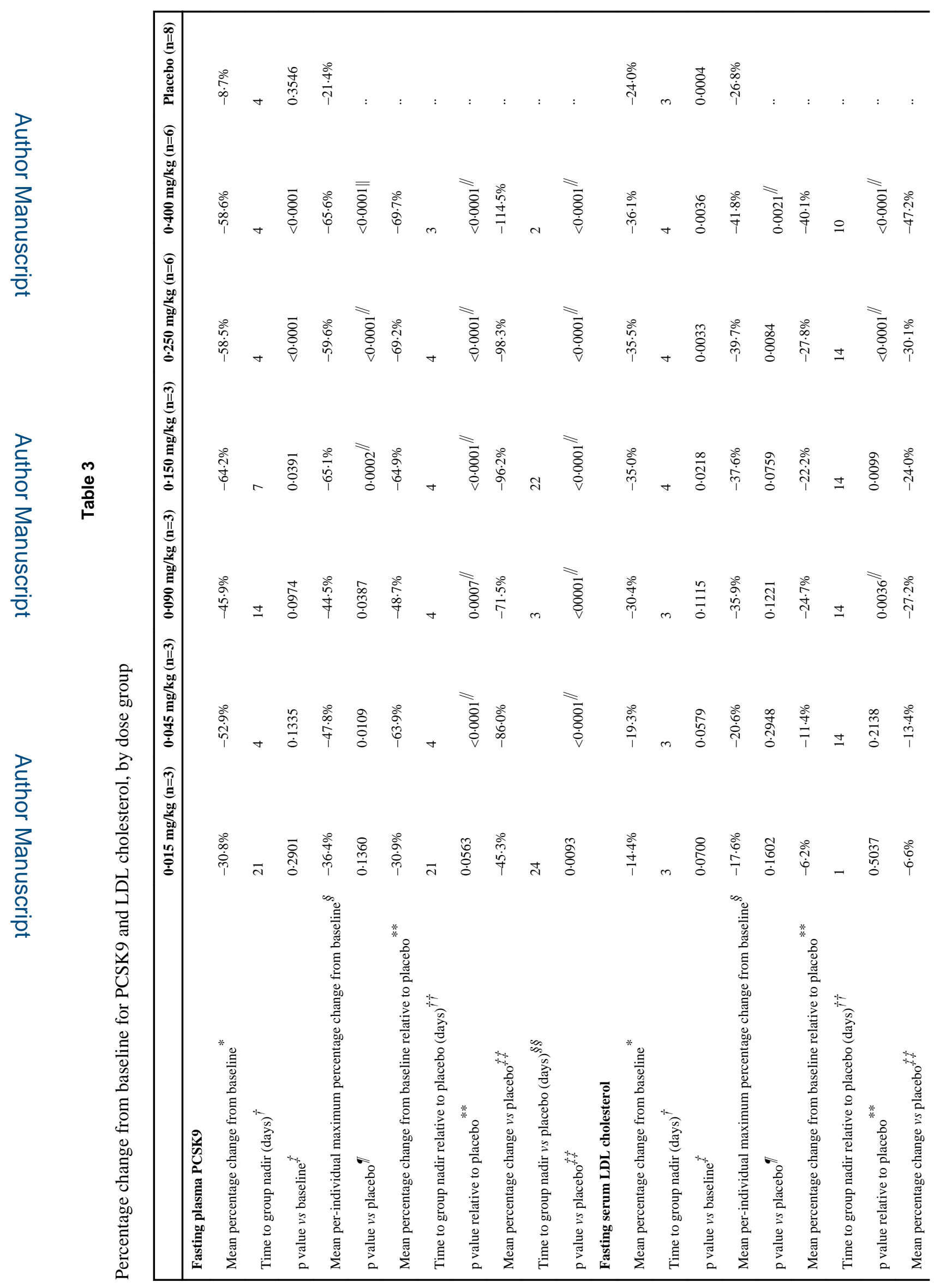




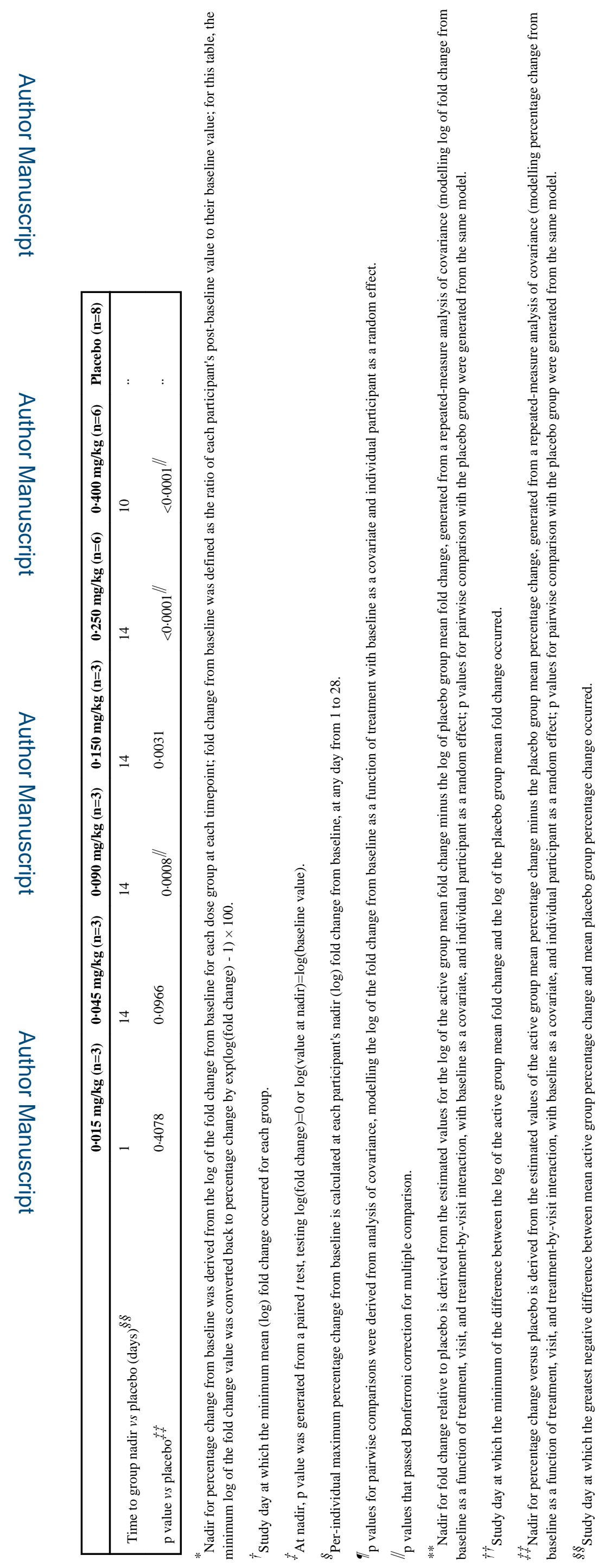

